\title{
Prostate-Specific Membrane Antigen PET/CT Combined with Sentinel Node Biopsy for Primary Lymph Node Staging in Prostate Cancer
}

\author{
Florentien J. Hinsenveld ${ }^{1}$, Esther M.K. Wit ${ }^{1}$, Pim J. van Leeuwen ${ }^{1}$, Oscar R. Brouwer ${ }^{1,2}$, Maarten L. Donswijk ${ }^{3}$, \\ Corinne N. Tillier ${ }^{1}$, Erik Vegt ${ }^{3}$, Erik van Muilekom ${ }^{1}$, Matthias N. van Oosterom ${ }^{1,2}$, Fijs W.B. van Leeuwen ${ }^{1,2}$, \\ and Henk G. van der Poel ${ }^{1}$
}

${ }^{1}$ Department of Urology, The Netherlands Cancer Institute, Amsterdam, The Netherlands; ${ }^{2}$ Interventional Molecular Imaging Laboratory, Department of Radiology, Leiden University Medical Center, Leiden, The Netherlands; and ${ }^{3}$ Department of Nuclear Medicine, The Netherlands Cancer Institute, Amsterdam, The Netherlands

\begin{abstract}
Our objective was to determine the diagnostic capabilities of combined prostate-specific membrane antigen (PSMA) PET/CT and sentinel node (SN) biopsy in PSMA PET/CT-negative patients for primary lymph node $(\mathrm{LN})$ staging in prostate cancer $(\mathrm{PCa})$ patients. Methods: Between January 2017 and March 2019, retrospectively, all consecutive patients with diagnosed intermediate- or high-risk primary PCa who underwent preoperative PSMA PET/CT ( ${ }^{68} \mathrm{Ga}$ or ${ }^{18} \mathrm{~F}-$ DCFPyL) followed by robot-assisted radical prostatectomy and extended pelvic LN dissection (ePLND) were included. All patients without suspected LN metastases on PSMA PET/CT were considered candidates for SN biopsy with indocyanine green-99mTc-nanocolloid or $99 \mathrm{mTc}$-nanocolloid with free indocyanine green used as tracers. The ePLND was used as a reference standard. Results: Of 53 patients, 22 had positive PSMA PET/CT results and 31 underwent subsequent SN biopsy after negative PSMA PET/CT results. In total, 23 patients (43\%) were pN1, of whom $6(26 \%)$ had negative PSMA PET/CT results and underwent subsequent SN biopsy. The combined use of SN biopsy and PSMA PET/CT identified all pN1 patients (100\% sensitivity; 95\% confidence interval, $86 \%-100 \%)$ and performed correct nodal staging in 50 of 53 patients $(94 \%$ diagnostic accuracy; 95\% confidence interval, 84\%-99\%). SN biopsy identified significantly smaller LN metastases (median diameter, $2.0 \mathrm{~mm}$; interquartile range, 1.0-3.8 mm) than PSMA PET/CT (median diameter, $5.5 \mathrm{~mm}$; interquartile range, 2.6-9.3 mm; $P=0.007$ ). Conclusion: Combining both modalities led to a $94 \%$ accuracy for nodal staging in diagnosed intermediate- and high-risk primary $\mathrm{PCa}$. Adding SN biopsy in patients with negative PSMA PET/CT results increased the combined sensitivity to $100 \%$ for detecting nodal metastases at ePLND. This diagnostic accuracy may provide valuable information for directing further treatment in $\mathrm{PCa}$ patients, such as the use of PSMA PET/CT and SN biopsy rather than EPLND as the preferred approach for staging before radiotherapy.
\end{abstract}

\footnotetext{
Received Jun. 6, 2019; revision accepted Aug. 29, 2019.

For correspondence or reprints contact: Florentien J. Hinsenveld, The Netherlands Cancer Institute, Woubruggestraat 13-1, Amsterdam, The Netherlands, 1059VN.

E-mail: florentienh@gmail.com

Published online Sep. 27, 2019.

COPYRIGHT (C 2020 by the Society of Nuclear Medicine and Molecular Imaging.
}

Key Words: prostate cancer; lymphatic metastasis; PSMA; sentinel node biopsy; image-guided surgery

J Nucl Med 2020; 61:540-545

DOI: 10.2967/jnumed.119.232199

\section{$\mathbf{P}$}

rostate cancer $(\mathrm{PCa})$ is one of the most frequently occurring malignancies and the third leading cause of cancer-related deaths in men (1). Because the presence of lymph node ( $\mathrm{LN})$ metastases has a major impact on patient management and prognosis, it is recommended that nodal sampling be performed for patients with a nomogram-assessed risk of over 5\% for LN metastases (Briganti or Memorial Sloan Kettering Cancer Centre nomogram) (2,3). Extended pelvic LN dissection (ePLND) is the gold standard for LN staging in PCa but has high morbidity, with a $20 \%$ incidence of complications such as infected lymphoceles and lymphedema $(4,5)$.

Theoretically, noninvasive imaging may enable preoperative identification of LNs or distant metastases, reducing the need for invasive staging with ePLND. Unfortunately, the diagnostic capabilities of conventional morphologic imaging such as CT and MRI for detecting LN metastases are limited (sensitivity of $42 \%$ and $39 \%$, respectively) (6). Molecular imaging technologies, such as the targeting of prostate-specific membrane antigen (PSMA) with PET/ CT radiotracers, have higher sensitivity and, especially, higher specificity for identifying LN metastases than morphologic imaging (7). PSMA PET/CT has demonstrated its advantage in restaging patients with biochemical recurrence after prostatectomy and has emerged as a potential tool for LN staging in the primary setting. Unfortunately, the first published results for PSMA PET/CT in primary staging indicated that its sensitivity for the detection of LN metastases is only moderate $(33 \%-66 \%)(8-10)$. This inaccuracy is partly due to the physical inability of PET to detect micrometastases $(<2 \mathrm{~mm})(11)$. In addition, PSMA PET/CT has a poor detection rate for metastases measuring between 2 and $4 \mathrm{~mm}$, since most current clinical PET/CT systems are still limited by a spatial resolution of 4-5 mm $(11,12)$. Therefore, PSMA PET/CT misses micrometastases that would be resected when ePLND is used as a staging method. Nevertheless, PSMA PET/CT has shown a high specificity (95\%-100\%) and positive predictive value (90\%-100\%) for $\mathrm{LN}$ metastases (8-10). Thus, although PSMA PET/CT may not be suitable 
for proving the absence of LN metastases, it can prove the presence of LN metastases in some patients.

Lymphatic mapping followed by sentinel node ( $\mathrm{SN}$ ) biopsy is considered standard practice in breast cancer, penile cancer, and melanoma and was successfully translated into PCa patients (13). Lymphatic mapping is based on the concept that the SN is the first draining LN of a tumor and therefore most likely to first contain metastatic cells. SN biopsy has demonstrated its value in the detection of LN metastases in intermediate- and high-risk PCa patients (sensitivity, 95\%) (14). A retrospective analysis indicated it has the potential to improve biochemical recurrence rates when combined with ePLND (15). One of the main advantages of combining SN biopsy with ePLND is that it helps identify aberrant tumor-draining LNs outside the field of ePLND. Because SN biopsy does enable the detection of micrometastases at histopathology, it could help identify LN metastases in PSMA PET/CT-negative patients (16).

In line with our earlier report, we hypothesized that combining the specificity of PSMA PET/CT with the sensitivity of SN biopsy could help optimize LN staging in primary PCa patients (16). Therefore, the objective of this study was to investigate, in primary LN staging of intermediate- to high-risk PSMA PET/CT PCa patients, the diagnostic accuracy of PSMA PET/CT combined with SN biopsy in PSMA PET/CT-negative patients, using an ePLND as reference standard.

\section{MATERIALS AND METHODS}

\section{Study Population}

In this retrospective single-center study, we consecutively included all patients with biopsy-proven intermediate- or high-risk PCa who underwent preoperative PSMA PET/CT followed by robot-assisted radical prostatectomy (RARP) with ePLND between January 2017 and March 2019. Patients without suggestive LNs on PSMA PET/CT imaging were considered candidates for an additional SN biopsy in a prospective SN biopsy study. The clinical T-stage was assessed using digital rectal examination and transrectal ultrasound and was classified according to the 2009 TNM staging system. All men underwent RARP combined with ePLND based on a nomogram-estimated risk of over $5 \%$ for LN metastases, assessed in the updated Briganti nomogram (2). Patients with androgen-deprivation therapy or patients with negative PSMA PET/CT not willing to undergo SN biopsy were excluded from this study. Patients with distant metastases (M1a/b) were not considered candidates for RARP and were therefore not included in this study. The study was approved by the institutional review board, and the need for written informed consent was waived.

\section{PSMA PET/CT}

PET/CT imaging was performed on a Gemini TF-II or TF Big Bore PET/CT scanner (Philips) starting with a dose-modulated low-dose CT scan (40 mAs, 2-mm reconstruction) from the proximal femora to the skull base, immediately followed by PET imaging of the same region. To improve urinary clearance of the tracer, $10 \mathrm{mg}$ of furosemide were intravenously administered. ${ }^{68} \mathrm{Ga}$-PSMA-11 was produced on-site compliant with the good-manufacturing-practice regulations. ${ }^{68} \mathrm{Ga}$ was obtained from a ${ }^{68} \mathrm{Ge} /{ }^{68} \mathrm{Ga}$ radionuclide generator and applied for radiolabeling of PSMA using an automated radiopharmacy cassette (Scintomics $\mathrm{GmbH}$ ). An intravenous bolus injection (100-MBq fixed dose) of the tracer was administered. As an alternative to ${ }^{68} \mathrm{Ga}$-PSMA-11, ${ }^{18}$ F-DCFPyL (2-(3-\{1-carboxy-5-[(6- ${ }^{18}$ F-fluoro-pyridine-3-carbonyl)amino]-pentyl\}-ureido)-pentanedioic acid) was used, a second-generation ${ }^{18}$ F-labeled small-molecule PSMA inhibitor with comparable results (17). PET/CT images were classified for LN staging according to their original reports: PSMA NO (negative) or PSMA N1 (positive).

\section{Lymphatic Mapping}

Patients who underwent SN biopsy were participants in a randomized phase II trial comparing the hybrid tracer indocyanine green (ICG)- ${ }^{99 \mathrm{~m}} \mathrm{Tc}$-nanocolloid to ${ }^{99 \mathrm{~m}} \mathrm{Tc}$-nanocolloid with free ICG for SN detection in $\mathrm{PCa}$, registered as the M13PSN study with registration number NL46580.031.13. ICG-99m Tc-nanocolloid or ${ }^{99 \mathrm{~m}} \mathrm{Tc}$-nanocolloid ( $2 \mathrm{~mL}, 240 \mathrm{MBq}$ ) was transrectally injected into the peripheral zone of each quadrant of the prostate under ultrasound guidance $4 \mathrm{~h}$ before surgery. To generate a roadmap for intraoperative localization of SNs, static planar lymphoscintigraphy of the pelvic area was performed at $15 \mathrm{~min}$ and $2 \mathrm{~h}$ after injection followed by a SPECT and low-dose CT scan (Symbia T; Siemens Healthcare). Patients receiving only ${ }^{99 \mathrm{~m}} \mathrm{Tc}$-nanocolloid were given an additional transperineal injection of free ICG $(5 \mathrm{mg}$ in $2 \mathrm{~mL}$ of water) 5 min before surgery to enable additional fluorescence guidance during surgery. Intraoperatively, SNs were localized using the Firefly (fluorescence) camera of the da Vinci Si surgical system (Intuitive) and a laparoscopic $\gamma$-probe (Europrobe 2; Eurorad). Ex vivo analysis of excised tissues was used to validate in vivo findings.

All PET/CT and lymphatic mapping images were viewed and reported using an Osirix Dicom Viewer (Pixmeo) and interpreted on the basis of clinical reports by nuclear medicine physicians with experience in PCa PET/CT imaging or lymphatic mapping.

\section{ePLND}

After SN biopsy, a complementary ePLND was performed, followed by RARP. The ePLND was defined as removal of nodes from the bifurcation of the common iliac artery up to the ureteral crossing (fossa of Marcille), along the external iliac vessels (the distal limit being the deep circumflex vein and femoral canal), the internal iliac vessels, and the obturator fossa. The lateral border was the genitofemoral nerve and the perivesical fat medially. The obtained specimens were mapped according to their anatomic side and region.

\section{Histologic Examination}

Specimens were sent for histopathologic examination according to International Society of Urological Pathology protocols. Prostatectomy specimens were formalin-fixed, paraffin-embedded, and classified according to the 2009 TNM classification for staging of PCa. LN tissue was examined by palpation, visual inspection, and sectioning. Both LNs and SNs were formalin-fixed, cut at $2 \mathrm{~mm}$, and paraffin-embedded. LN

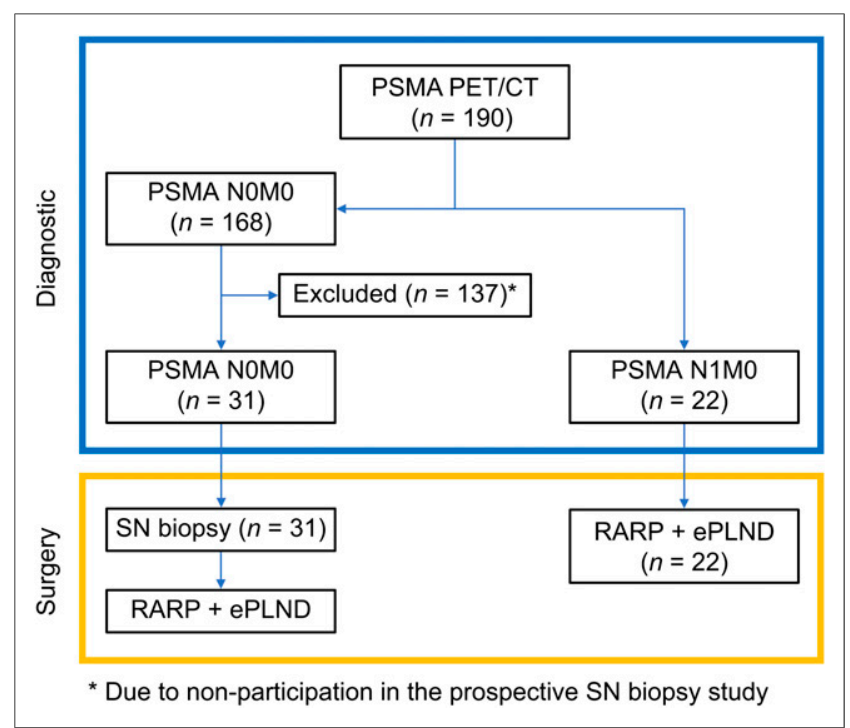

FIGURE 1. Flowchart for group distribution. 
sections were stained with hematoxylin and eosin, whereas SNs were first cut at 3 levels (150- $\mu \mathrm{m}$ intervals). A CAM5.2 antibody was used for immunohistochemical analysis (catalog no. 345779; Becton Dickinson Biosciences). Disease positivity was defined as the presence of any metastatic deposit of PCa in the LNs examined, and the diameter, expressed in millimeters, was recorded. All pathologists had access to clinical reports.

\section{Statistical Analysis}

To compare clinicopathologic characteristics, the independent $t$ test and Mann-Whitney test (means and medians, respectively, from continuous data) and the $\chi^{2}$ test and Fisher exact test (categoric data) were used. To depict the diagnostic accuracy of combined PSMA PET/CT and SN biopsy on a per-patient basis, $2 \times 2$ tables were used. Positive matching was defined as correlation between test positivity and histologic positivity. Histopathologic analysis of the LNs was used as the reference standard. PSMA PET/CT was repeated in all patients with a false-positive scan result (i.e., a positive LN on PSMA PET/CT but no positive LN found on histopathology) and any detectable prostate-specific antigen (PSA) after surgery. All statistical tests were 2-sided, and differences were considered statistically significant when the $P$ value was less than 0.05 . Statistical analysis was performed with SPSS Statistics, version 24.0 (IBM).

\section{RESULTS}

\section{Preoperative Characteristics}

In total, 190 patients who underwent PSMA PET/CT were candidates for this study. Of those, 168 had negative PSMA PET/CT results (PSMA NOM0) and 22 had positive results (PSMA N1M0). Ultimately, 53 patients who had undergone preoperative PSMA PET/CT with ${ }^{68} \mathrm{Ga}$-PSMA-11 $(n=46)$ or ${ }^{18} \mathrm{~F}-\mathrm{DCFPyL}(n=7)$ were included, because 31 patients with negative PSMA PET/CT results agreed to join the prospective study on SN biopsy (Fig. 1; Table 1). The main reason for nonparticipation $(n=137)$ was the unwillingness of the patient to undergo intraprostatic tracer injections for lymphatic mapping. LN hotspots on PSMA PET/CT were located in the external iliac $(n=12)$, internal iliac $(n=2)$, obturator fossa $(n=10)$, presacral $(n=2)$, and pararectal $(n=$ 1) regions. One patient with negative PSMA PET/CT results had no PSMA tracer accumulation in the primary tumor. During lymphatic mapping for SN biopsy, 17 patients (55\%) were injected with ICG-99m Tc-nanocolloid, and in 14 patients $(45 \%){ }^{99 \mathrm{~m}} \mathrm{Tc}-$ nanocolloid was used with free ICG injected just before surgery. Eight patients $(26 \%)$ had unilateral visualization of SNs on SPECT/CT instead of bilateral $(n=23 / 31,74 \%)$. Four preoperatively identified SNs were deemed technically irresectable because of inaccessible locations (presacral and pararectal).

\section{Pathology of ePLND and SN Biopsy}

In total, 23 of 53 patients $(43 \%)$ had $\mathrm{LN}$ metastases at histopathologic evaluation of ePLND (Table 2). Positive LNs $(n=$ 66 , including SNs) were located in the external iliac $(n=21)$, obturator fossa $(n=37)$, fossa of Marcille $(n=2)$, preprostatic $(n=1)$, pararectal $(n=1)$, and presacral $(n=4)$ regions. SN biopsy identified

TABLE 1

Baseline Characteristics

\begin{tabular}{|c|c|c|c|c|}
\hline Characteristic & Total & PSMA N1M0 & PSMA NOMO + SN biopsy & $P$ \\
\hline Patients $(n)$ & 53 & 22 & 31 & \\
\hline \multicolumn{5}{|l|}{ Age $(y)$} \\
\hline Mean & 67 & 67 & 67 & 0.744 \\
\hline $95 \%$ confidence interval & $65-69$ & $64-70$ & $65-70$ & \\
\hline \multicolumn{5}{|l|}{ PSA (ng/mL) } \\
\hline Median & 11 & 11 & 10 & 0.362 \\
\hline IQR & $8-20$ & $9-20$ & $8-21$ & \\
\hline Clinical stage $(n)$ & & & & 0.166 \\
\hline $\mathrm{T} 1$ & $4(8 \%)$ & $2(9 \%)$ & $2(7 \%)$ & \\
\hline $\mathrm{T} 2$ & 27 (51\%) & $8(36 \%)$ & $19(61 \%)$ & \\
\hline T3 & $22(42 \%)$ & $12(55 \%)$ & $10(32 \%)$ & \\
\hline Clinical ISUP grade $(n)$ & & & & 0.286 \\
\hline 1 & $2(4 \%)$ & $2(9 \%)$ & 0 & \\
\hline 2 & $9(17 \%)$ & $2(9 \%)$ & $7(23 \%)$ & \\
\hline 3 & $10(19 \%)$ & $5(23 \%)$ & $5(16 \%)$ & \\
\hline 4 & $19(36 \%)$ & $9(41 \%)$ & $10(32 \%)$ & \\
\hline 5 & $13(25 \%)$ & $4(18 \%)$ & $9(29 \%)$ & \\
\hline D’Amico classification ( $n$ ) & & & & 1.000 \\
\hline Intermediate-risk & $8(15 \%)$ & $2(9 \%)$ & $6(19 \%)$ & \\
\hline High-risk & $45(85 \%)$ & $20(91 \%)$ & $25(81 \%)$ & \\
\hline \multicolumn{5}{|c|}{ Risk of LN metastases (Briganti) (\%) } \\
\hline Median & 29 & 26 & 29 & 0.391 \\
\hline IQR & $23-54$ & $23-52$ & $24-61$ & \\
\hline
\end{tabular}

ISUP $=$ International Society of Urological Pathology. 
TABLE 2

Pathologic Outcomes

\begin{tabular}{|c|c|c|c|c|}
\hline Outcome & Total & PSMA N1M0 & PSMA NOMO + SN biopsy & $P$ \\
\hline Patients $(n)$ & 53 & 22 & 31 & \\
\hline Pathologic stage $(n)$ & & & & 0.061 \\
\hline $\mathrm{T} 2$ & $19(36 \%)$ & $4(18 \%)$ & $15(48 \%)$ & \\
\hline T3a & $17(32 \%)$ & $8(36 \%)$ & $9(29 \%)$ & \\
\hline T3b & $17(32 \%)$ & $10(46 \%)$ & $7(23 \%)$ & \\
\hline Pathologic ISUP grade $(n)$ & & & & 0.819 \\
\hline 2 & $11(21 \%)$ & $5(23 \%)$ & $6(19 \%)$ & \\
\hline 3 & $24(45 \%)$ & $11(50 \%)$ & $13(42 \%)$ & \\
\hline 4 & $5(9 \%)$ & $1(\% 5)$ & $4(13 \%)$ & \\
\hline 5 & $13(25 \%)$ & $5(23 \%)$ & $8(26 \%)$ & \\
\hline \multicolumn{5}{|l|}{ LNs removed (including SNs) } \\
\hline$n$ & 1,168 & 475 & 693 & \\
\hline Per patient, mean & 22 & 22 & 22 & 0.752 \\
\hline Per patient, 95\% confidence interval & $20-25$ & $19-25$ & $19-26$ & \\
\hline Positive LNs & & & & $<0.001$ \\
\hline$n$ & 66 & 52 & 14 & \\
\hline$\%$ of total LNs & $6 \%$ & $11 \%$ & $2 \%$ & \\
\hline \multicolumn{5}{|l|}{ SNs removed } \\
\hline$n$ & & & 230 & \\
\hline Per patient, median & & & 7 & \\
\hline Per patient, IQR & & & $2-9$ & \\
\hline \multicolumn{5}{|l|}{ Positive SNs } \\
\hline$n$ & & & 10 & \\
\hline$\%$ of total SNs & & & $4 \%$ & \\
\hline pN1 patients $(n)$ & $23(43 \%)$ & 17 (77\%) & $6(19 \%)$ & $<0.001$ \\
\hline
\end{tabular}

significantly smaller $\mathrm{LN}$ metastases (median diameter, $2.0 \mathrm{~mm}$; interquartile range [IQR], 1.0-3.8 mm) than did PSMA PET/CT (median diameter, $5.5 \mathrm{~mm}$; IQR, 2.6-9.3 mm; $P=0.007$ ). The smallest diameter for LN metastases detected with PSMA PET/CT was 2.0 $\mathrm{mm}$. SN biopsy detected a LN with only isolated tumor cells.

Evaluating the pathologic outcomes of SN biopsy separately, we found that SNs were the only tumor-containing LNs in 4 of 6 pN1 patients $(67 \%)$. SNs outside the standard ePLND template (presacral and pararectal regions) were identified and resected in 7 cases, none of which harbored metastatic deposits. In these cases, SNs inside the standard ePLND field were also resected. All patients with negative SNs had no metastases in the ePLND field; thus, no false-negative SN biopsies were seen.

Among the PSMA PET/CT-positive patients, 2 of the 5 falsepositive patients had a detectable PSA 4 mo after surgery, and repeated PSMA PET/CT showed a suggestive LN similar to the initial imaging in the obturator and internal iliac region. This finding suggests an incomplete ePLND and possible correct staging by PSMA $\mathrm{PET} / \mathrm{CT}$. Consequently, these 2 patients were staged as true-positive. As a result, the positive predictive value of PSMA PET/CT was $86 \%$ (19/22 patients) in this study (Fig. 2).

In 9 of 19 patients $(47 \%)$ with true-positive PSMA PET/CT results, the histopathology of ePLND revealed additional LN metastases undetected by PSMA PET/CT. In one case, PSMA PET/CT identified a histologically positive LN with a diameter of $7.0 \mathrm{~mm}$ outside the standard ePLND field (pararectal region). This LN metastasis was the only metastatic node in this patient and would not have been resected if preoperative PSMA PET/CT had not been performed.

\section{Performance of Combined PSMA PET/CT and SN Biopsy}

PSMA PET/CT detected 17 of 23 (74\%) pN1 patients. Subsequently, SN biopsy correctly staged the remaining $6 \mathrm{pN} 1$ patients with false-negative PSMA PET/CT results, leading to a combined sensitivity of $100 \%$ (95\% confidence interval, 86-100) (Fig. 2). Detected LN metastases by PSMA PET/CT $(52 / 66=$ $79 \%)$ were located in the external iliac $(n=17)$, obturator fossa $(n=27)$, fossa of Marcille $(n=2)$, preprostatic $(n=1)$, pararectal $(n=1)$, and presacral $(n=4)$ regions. Positive SNs were located in the external iliac $(n=4)$ and obturator fossa $(n=6)$ regions. Correct LN staging was performed in 50 of 53 patients, resulting in 94\% diagnostic accuracy (95\% confidence interval, 84\%-99\%) (Fig. 2). The 3 incorrectly staged patients had falsepositive PSMA PET/CT results (pN0 at ePLND and undetectable PSA after surgery). These cases had suspected LNs on PSMA PET/CT inside the ePLND field (obturator fossa and external iliac) 


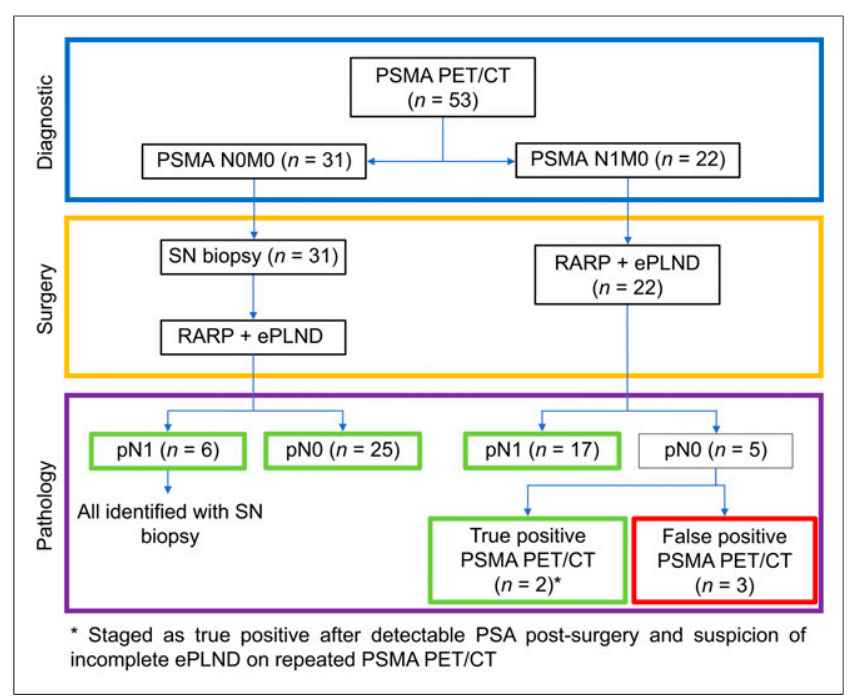

FIGURE 2. Evaluation of combined PSMA PET/CT and SN biopsy for detection of LN metastases. Green frame indicates correct LN staging; red frame indicates incorrect $L N$ staging.

and were regarded as positive, based on LN morphology (sizes 4-10 mm) and PSMA expression (all scored 1) according to PROMISE criteria, and were clinically staged as N1 (18). In those patients, no biochemical recurrence was detected during follow-up (4-12 mo).

\section{DISCUSSION}

\section{Principal Findings}

In patients with negative PSMA PET/CT results, the addition of SN biopsy managed to correctly stage all patients with falsenegative PSMA PET/CT results, with EPLND as a reference standard. As such, this creates a best-of-both-worlds scenario combining the high specificity of PSMA PET/CT with the high sensitivity of SN biopsy for detecting LN metastases in patients with intermediate- or high-risk PCa, resulting in $100 \%$ combined sensitivity ( $n=23 \mathrm{pN} 1$ patients) and correct nodal staging in $94 \%(n=50 / 53)$ of cases. SN biopsy is able to detect significantly smaller LN metastases (median diameter, $2.0 \mathrm{~mm}$; IQR, 1.0-3.8 mm) than can PSMA PET/CT (median diameter, $5.5 \mathrm{~mm}$; IQR, 2.6-9.3 $\mathrm{mm}$; $P=0.007$ ).

\section{Combining PSMA PET/CT and SN Biopsy}

In addition to being used for primary LN staging, PSMA PET/ CT was used to select men for SN biopsy in addition to ePLND. $\mathrm{SN}$ biopsy can play a role in identifying small $\mathrm{LN}$ metastases with high sensitivity in men without suggestive LNs on preoperative imaging, as demonstrated in the present scenario. SN biopsy accurately identified all $6 \mathrm{pN} 1$ patients with negative PSMA PET/ CT results, with ePLND as a reference standard. Studies examining the sensitivity of SN biopsy reported rates of $78 \%-100 \%$, and a systematic review showed an overall mean sensitivity of $95.2 \%$ (IQR, 81.8\%-100\%) (14,19-22). The combination of SN biopsy and PSMA PET/CT led to a combined sensitivity of $100 \%$ and therefore seems to be a very accurate diagnostic combination for identifying LN involvement at primary staging of PCa. Both modalities combined can differentiate between $\mathrm{pN} 0$ and $\mathrm{pN} 1$ patients with 94\% accuracy. This high accuracy cannot replace the ePLND in patients receiving RARP, since many $\mathrm{LN}$ metastases are left behind if no ePLND is performed in addition to positive SN biopsy, and secondary surgery for the remaining LNs is not preferable (14). However, this high diagnostic accuracy of the combined approach might replace the ePLND in patients receiving radiotherapy as curative treatment, since additional radiotherapy is given on the ePLND field when SNs are positive. In these patients, combining PSMA PET/ CT and SN biopsy serves as a less invasive LN staging method before curative treatment.

In this study, we identified a case in which PSMA PET/CT detected a LN metastasis in the pararectal region with a negative ePLND. This LN metastasis would have been left behind without PSMA PET/CT. No tumor-positive SNs were found outside the standard ePLND, regardless of the outcome of ePLND. However, Wit et al. reported a rate of $4.9 \%$ (IQR, $0 \%-10.2 \%$ ) of such cases, indicating that for 1 in 20 patients a LN metastasis would have been left behind without SN biopsy (14).

The significant difference between the diameter of detected LN metastases by PSMA PET/CT or SN biopsy is in line with our recent review in which we suggested combining different imageguided surgery techniques for identifying different types of LN metastases (16). The thorough histopathologic evaluation of SNs can result in a high chance of detecting micrometastases. With PET/CT systems, an intense PSMA signal is required to detect metastatic deposits below the spatial resolution of 4-5 mm (12). ${ }^{18}$ F-based PSMA tracers have a lower positron penetration depth than does ${ }^{68} \mathrm{Ga}$, potentially resulting in a better spatial resolution (23). This advantage seems limited in soft tissues but may become more important with newer, high-resolution PET/CT systems (24).

PSMA PET/CT can identify LN metastases with high specificity, as seen in previous studies reporting specificity rates of $95 \%-99 \%$, and is currently the best available imaging tool in the management of PCa $(8,9)$. As such, PSMA PET/CT offers valuable prognostic information on staging before RARP. In a previous study, only $50 \%$ of men with LN hotspots on PSMA PET/CT experienced a complete biochemical response to RARP, despite an ePLND (25). By contrast, $83.3 \%$ of men with negative PSMA PET/CT results had a complete biochemical response after RARP. The PSMA PET/CT results were more predictive of biochemical response to RARP than established clinical predictors such as preoperative PSA level, Gleason score, and clinical $\mathrm{T}$ stage. This finding suggests that PSMA PET/CT can be used to select patients who should be considered to receive multimodality treatments rather than surgery alone and has the potential to become an important instrument in the future management of newly diagnosed PCa.

The specificity of PSMA PET/CT seems to be influenced by the quality and completeness of the ePLND, since an incomplete ePLND results in higher false-positive rates. In the present study, 2 cases with suspected incomplete ePLND were also shown (detectable PSA after surgery and suggestive LNs on both preoperative and postoperative PSMA PET/CT, despite pN0 at ePLND). These missed suspected LN metastases were located in technically accessible locations, and it is therefore interesting to speculate whether image-guided surgery, such as SN biopsy or ${ }^{99 \mathrm{~m}}$ Tc-PSMA targeted surgery, would have resected these LNs. Additionally, if SN biopsy had been performed in PSMA PET/CT-positive patients, LN metastases in the 3 falsepositive PSMA PET/CT patients would potentially have been detected, especially considering that all false-positive LNs were regarded as positive according to the PROMISE criteria (18).

\section{Clinical Implications}

Our findings may have several clinical implications. First, earlier observations suggested that adding SN biopsy to ePLND improved 
biochemical recurrence-free survival $(14,15)$. Data from the current study support that the value of SN biopsy may be limited in men with positive PSMA PET/CT results. Second, our data discourage the use of ePLND for staging purposes in men before radiotherapy for PCa. In these patients, the high specificity of PSMA PET/CT obviates a diagnostic ePLND in men with a positive scan. In radiotherapy patients with negative PSMA PET/CT results, SN biopsy rather than ePLND is to be the preferred diagnostic approach, considering the lower morbidity of the former (14).

\section{Strengths and Limitations}

The main strength of this study is the evaluation of the combination of both PSMA PET/CT and SN biopsy as a new diagnostic tool for the detection of LN metastases validated by ePLND, resulting in better primary staging of PCa patients and complementing our earlier report (16). Nevertheless, there are several significant limitations to this study. First, this study was a retrospective analysis. Second, the sample size was small because of exclusion of PSMA PET/CT-negative patients without SN biopsy. Possibly because of a limited number of SN-biopsy participants harboring LN metastases, we could not detect an additional value for SN biopsy over ePLND in terms of LN staging, since no patients had positive SNs outside the ePLND field. Third, because of limited follow-up data, it was not possible to analyze whether long-term biochemical recurrence occurred in $\mathrm{pN} 0$ patients.

\section{CONCLUSION}

Combining preoperative PSMA PET/CT and, if PET/CT is negative, SN biopsy led to a high accuracy of $94 \%$ for LN staging in primary staging of intermediate- and high-risk PCa in the current study. Adding SN biopsy for patients with negative PSMA PET/CT results increases the combined sensitivity to $100 \%$ for detecting nodal metastases at ePLND. This diagnostic accuracy may provide valuable information for directing further treatment in PCa patients, such as PSMA PET/CT and SN biopsy rather than ePLND as the preferred approach for staging before radiotherapy. Yet, prospective studies with larger sample sizes and a longer followup are required to substantiate these findings.

\section{DISCLOSURE}

No potential conflict of interest relevant to this article was reported.

\section{KEY POINTS}

QUESTION: Does combined PSMA PET/CT and SN biopsy provide good diagnostic accuracy for primary $L N$ staging in $\mathrm{PCa}$ ?

PERTINENT FINDINGS: In this retrospective cohort study of 53 intermediate- and high-risk PCa patients, a $100 \%$ sensitivity and 94\% diagnostic accuracy for primary LN staging was achieved when PSMA PET/CT was combined with SN biopsy in PSMA PET/CT-negative patients.

IMPLICATIONS FOR PATIENT CARE: This high diagnostic accuracy may provide valuable information for directing further treatment in PCa patients, such as PSMA PET/CT and SN biopsy rather than ePLND as the preferred approach for staging before radiotherapy.

\section{REFERENCES}

1. Torre LA, Bray F, Siegel R, Ferlay J, Lortet-Tieulent J, Jemal A. Global cancer statistics, 2012. CA Cancer J Clin. 2015;65:87-108.

2. Briganti A, Larcher A, Abdollah F, et al. Updated nomogram predicting lymph node invasion in patients with prostate cancer undergoing extended pelvic lymph node dissection: the essential importance of percentage of positive cores. Eur Urol. 2012;61:480-487.

3. Dynamic prostate cancer nomogram: coefficients. Memorial Sloan Kettering Cancer Center website. https://www.mskcc.org/nomograms/prostate/pre_op/coefficients. Updated April 10, 2018. Accessed November 19, 2019.

4. Briganti A, Blute ML, Eastham JA, et al. Pelvic lymph node dissection in prostate cancer. Eur Urol. 2009;55:1251-1265.

5. Mottet N, Bellmunt J, Bolla M, et al. EAU-ESTRO-SIOG guidelines on prostate cancer. Part 1: screening, diagnosis, and local treatment with curative intent. Eur Urol. 2017;71:618-629.

6. Hövels AM, Heesakkers RAM, Adang EM, et al. The diagnostic accuracy of CT and MRI in the staging of pelvic lymph nodes in patients with prostate cancer: a meta-analysis. Clin Radiol. 2008;63:387-395.

7. Hofman MS, Hicks RJ, Maurer T, Eiber M. Prostate-specific membrane antigen PET: clinical utility in prostate cancer, normal patterns, pearls, and pitfalls. Radiographics. 2018;38:200-217.

8. van Leeuwen PJ, Emmett L, Ho B, et al. Prospective evaluation of ${ }^{68}$ galliumprostate-specific membrane antigen positron emission tomography/computed tomography for preoperative lymph node staging in prostate cancer. BJU Int. 2017;119:209-215.

9. Maurer T, Gschwend JE, Rauscher I, et al. Diagnostic efficacy of ${ }^{68}$ galliumPSMA positron emission tomography compared to conventional imaging for lymph node staging of 130 consecutive patients with intermediate to high risk prostate cancer. J Urol. 2016;195:1436-1443.

10. Budäus L, Leyh-Bannurah S-R, Salomon G, et al. Initial experience of ${ }^{68} \mathrm{Ga}-$ PSMA PET/CT imaging in high-risk prostate cancer patients prior to radical prostatectomy. Eur Urol. 2016;69:393-396.

11. Jilg CA, Drendel V, Rischke HC, et al. Diagnostic accuracy of Ga-68-HBEDCC-PSMA-ligand-PET/CT before salvage lymph node dissection for recurrent prostate cancer. Theranostics. 2017;7:1770-1780.

12. Vandenberghe S, Mikhaylova E, D'Hoe E, Mollet P, Karp JS. Recent developments in time-of-flight PET. EJNMMI Phys. 2016;3:3.

13. Wawroschek F, Vogt H, Weckermann D, Wagner T, Harzmann R. The sentinel lymph node concept in prostate cancer: first results of gamma probe-guided sentinel lymph node identification. Eur Urol. 1999;36:595-600.

14. Wit EMK, Acar C, Grivas N, et al. Sentinel node procedure in prostate cancer: a systematic review to assess diagnostic accuracy. Eur Urol. 2017;71:596-605.

15. Grivas N, Wit E, Kuusk T, et al. The impact of adding sentinel node biopsy to extended pelvic lymph node dissection on the biochemical recurrence of prostate cancer patients treated with robot-assisted radical prostatectomy. J Nucl Med. 2018;59:204-209.

16. van Leeuwen FWB, Winter A, Van Der Poel HG, et al. Technologies for imageguided surgery for managing lymphatic metastases in prostate cancer. Nat Rev Urol. 2019;16:159-171.

17. Dietlein M, Kobe C, Kuhnert G, et al. Comparison of $\left[{ }^{18} \mathrm{~F}\right] \mathrm{DCFPyL}$ and $\left[{ }^{68} \mathrm{Ga}\right]$ Ga-PSMA-HBED-CC for PSMA-PET imaging in patients with relapsed prostate cancer. Mol Imaging Biol. 2015;17:575-584.

18. Eiber M, Herrmann K, Calais J, et al. Prostate cancer molecular imaging standardized evaluation (PROMISE): proposed miTNM classification for the interpretation of PSMA-ligand PET/CT. J Nucl Med. 2018;59:469-478.

19. Miki J, Yanagisawa T, Tsuzuki S, et al. Anatomical localization and clinical impact of sentinel lymph nodes based on patterns of pelvic lymphatic drainage in clinically localized prostate cancer. Prostate. 2018;78:419-425.

20. KleinJan GH, van den Berg NS, de Jong J, et al. Multimodal hybrid imaging agents for sentinel node mapping as a means to (re)connect nuclear medicine to advances made in robot-assisted surgery. Eur J Nucl Med Mol Imaging. 2016;43: $1278-1287$.

21. Harke NN, Godes M, Wagner C, et al. Fluorescence-supported lymphography and extended pelvic lymph node dissection in robot-assisted radical prostatectomy: a prospective, randomized trial. World J Urol. 2018;36: 1817-1823.

22. Ramírez-Backhaus M, Mira Moreno A, Gómez Ferrer A, et al. Indocyanine green guided pelvic lymph node dissection: an efficient technique to classify the lymph node status of patients with prostate cancer who underwent radical prostatectomy. J Urol. 2016;196:1429-1435.

23. Kesch C, Kratochwil C, Mier W, Kopka K, Giesel FL. ${ }^{68} \mathrm{Ga}$ or ${ }^{18} \mathrm{~F}$ for prostate cancer imaging? J Nucl Med. 2017;58:687-688.

24. Sánchez-Crespo A, Andreo P, Larsson SA. Positron flight in human tissues and its influence on PET image spatial resolution. Eur J Nucl Med Mol Imaging. 2004; 31:44-51.

25. van Leeuwen PJ, Donswijk M, Nandurkar R, et al. ${ }^{68} \mathrm{Ga}$ PSMA PET/CT predicts complete biochemical response from radical prostatectomy and lymph node dissection in intermediate and high-risk prostate cancer. BJU Int. 2019;124: 62-68. 